Gerión. Revista de Historia Antigua

ISSN: 0213-0181

\title{
Una sepoltura privilegiata a mosaico nella Basilica di Uppenna (Tunisia)
}

\author{
Luigi Quattrocchi ${ }^{1}$
}

Recibido: 13 de septiembre de 2017 / Aceptado: 27 de febrero de 2018

Riassunto. L'articolo si concentra su una sepoltura privilegiata nella Basilica di Uppenna, in Tunisia. Questa sepoltura fu probabilmente di un vescovo donatista che partecipò nel 411 alla Concilio di Cartagine. La peculiarità di questa sepoltura è la sovrapposizione di due mosaici funerari: uno più antico datato al secondo quarto del $\mathrm{V}$ secolo mentre il più recente datato nella seconda metà del $\mathrm{V}$ secolo. Il trattamento riservato a questa sepoltura è simile a quello di altre due sepolture vescovili della stessa basilici. Questi elementi, uniti ad altri, suggeriscono che questa sepoltura fosse riservata a un vescovo o comunque a un personaggio importante all'interno della comunità.

Parole chiave: paleocristiano; mosaico funerario; Uppenna; episcopus; donatismo.

\section{[en] A privileged burial in the Basilica of Uppenna (Tunisia)}

\begin{abstract}
The paper is about a privileged burial in the Basilica of Uppenna (Tunisia). This burial was probably for a Donatist bishop who participated to the Conference of Carthage (411). The peculiarity of this burial is that it consists in two overlapping tomb mosaics: the most ancient one dated to the second quarter of $5^{\text {th }}$ century and the most recent one dated to the second half of $5^{\text {th }}$ century. The treatment reserved for this burial is similar to that of two tombs reserved for bishops in the same basilica. All this, together with other elements, suggest that this burial was reserved for a bishop, or another élite person. Keywords: Early Christian; Tomb mosaic; Uppenna; Episcopus; Donatism.
\end{abstract}

Sommario. 1. Introduzione. 2. "La salle à l'est de la cuve baptisimale", vano XVI. 3. Le sepolture vescovili presenti a Uppenna. 4. Una basilica donatista? 5. Ipotesi sull'identificazione del defunto. 6. Conclusioni. 7. Bibliografia.

Cómo citar: Quattrocchi, L. (2018): Una sepoltura privilegiata a mosaico nella Basilica de Uppena (Tunisia), en Gerión 36/1, 95-108.

1 Universidad Carlos III de Madrid.

E-mail: 1.quattrocchi@hotmail.it 


\section{Introduzione}

La Basilica di Uppenna fu oggetto di scavi a più riprese a partire dal 1881 e proseguiti per tutto il secolo scorso. Ubicata nelle vicinanze dell'odierna Henchir Chigarnia, vicino alla costa orientale della Tunisia, la basilica possedeva tre navate e due absidi più numerose cappelle e due battisteri.

Bisogna segnalare che, per quanto questa basilica sia stata oggetto di studi, da parte di più studiosi, ${ }^{2}$ tra cui una monografia incentrata proprio sui mosaici funerari, ${ }^{3}$ mancano i dati di scavo e lo studio del materiale ritrovato. Le strutture sono datate, talvolta in maniera opinabile, ${ }^{4}$ sulla base dei mosaici ritrovati, datati a loro volta solo in maniera stilistica ${ }^{5}$ e anche in questo caso con qualche perplessità.

La cosiddetta salle à l'Est de la cuve baptismale, ovvero il vano XVI, conservava i resti della sepoltura oggetto di questo studio, in una zona lontana dal resto delle sepolture.

\section{2. "La salle à l'est de la cuve baptisimale", vano XVI}

Il vano XVI (Fig. 1), con misure di 3,23 x 4,09 m, era interamente occupato da un tessellato con una doppia cornice: la più esterna una semplice fila dentellata (DG 2j) ${ }^{6}$ e la più interna del tipo a fila di cerchi tangenti caricati da un cerchio composto di quattro fusi che formano un quadrato concavo delineato (DG 45a) che contornava la sepoltura e una composizione geometrica del tipo reticolato di fasce, non delineate, con quadrati negli incroci (DG $145 \mathrm{f}$ var.).

Questa composizione ha avuto rimaneggiamenti in epoca moderna ma dai quadrati superstiti possiamo capire che all'interno degli stessi erano presenti due decorazioni: nodi di Salomone con fiori di loto e croci. In questa composizione trovava spazio la sepoltura che presenta una particolarità: si tratta di una sepoltura a mosaico, come tante altre ritrovate all'interno della basilica, però organizzata in due tessellati, uno più antico e uno più recente, sovrapposti.

Il mosaico più antico (Fig. 2) è tripartito, misura $189 \times 64 \mathrm{~cm}$, con tessere dai colori bianco, nero, verde, ocra, presenta una semplice cornice a linee di tessere nere (DG 1a) con il pannello superiore decorato con triangoli negli angoli e una corona d'alloro nel centro che a sua volta contorna una croce monogrammata con le lettere alpha e omega; il pannello centrale presenta l'epitaffio in sette righe, con linee di supporto:

Nel pannello inferiore è presente una composizione del tipo a reticolato di quadrati curvilinei e quadrifoglio inscritto sulla diagonale come file di fusi (DG 131f var.).

Il mosaico più recente (Fig. 3) è tripartito, misura $206 \times 71 \mathrm{~cm}$, con tessere dai colori bianco, nero, rosso, bruno, rosa, verde, con cornice semplice (DG 1a), col pannello superiore decorato con fiori di loto ai lati e una corona dentellata, al cui interno troviamo un quadrato posto sulla punta; il pannello centrale è interessato dall'epitaffio in otto righe senza linea di supporto:

\footnotetext{
Raynal 1973, 33-72; Duval 1976, 97-119; Raynal 1982, 179-284, e 1995, 63-86.

Raynal 2005.

Stevens 2007, 689-694.

In questo lavoro le datazioni sono quelle utilizzate in Quattrocchi 2017, non quelle di Raynal 2005.

Per descrivere la decorazione geometrica verranno utilizzati i riferimenti al Balmelle et alii 1985 e 2002 (=DG).
} 


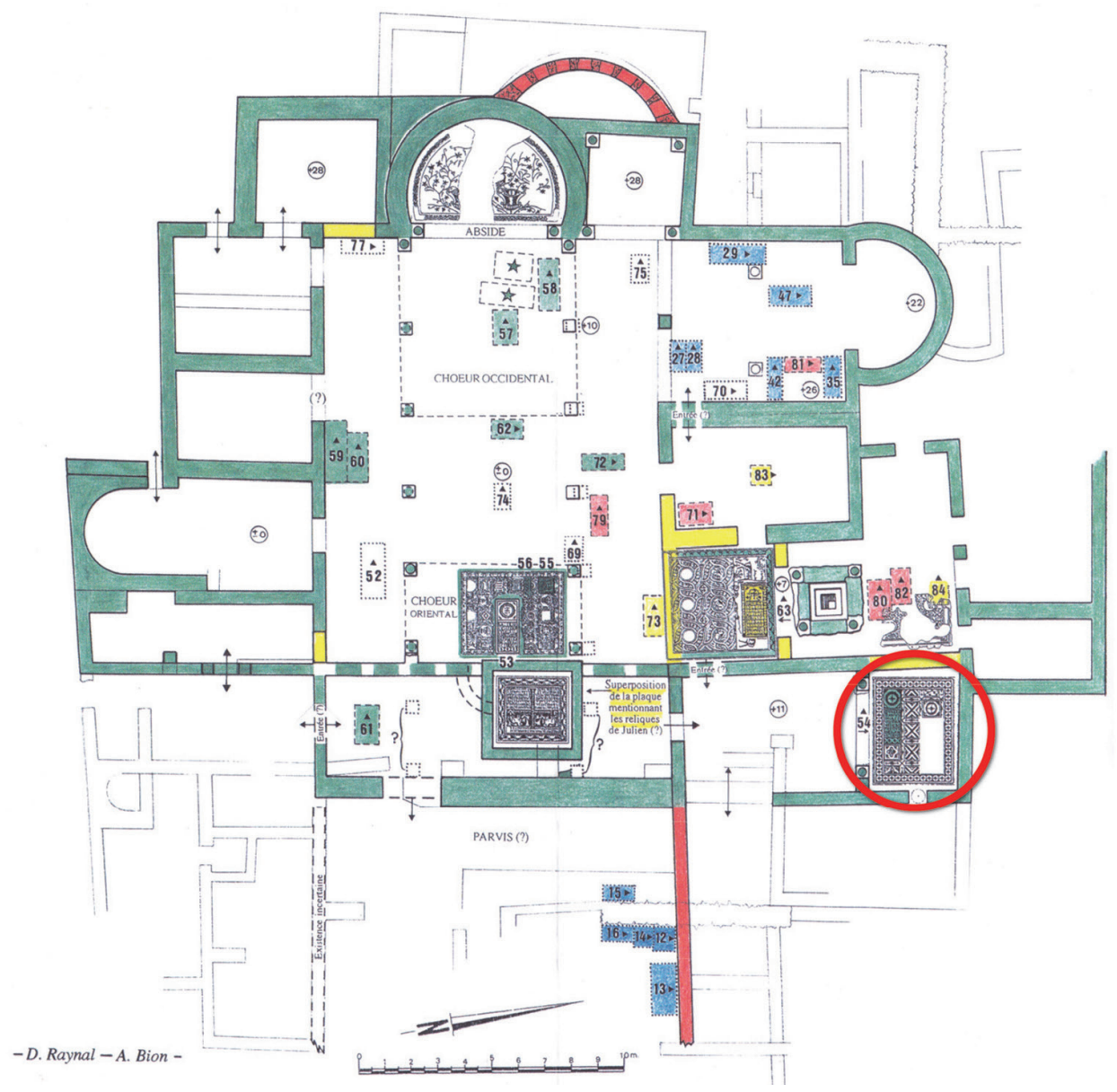

Figura 1. Basilica di Uppenna con indicazione del vano XVI (Raynal 2005, modificato dall'autore).

Il pannello inferiore, di forma rettangolare, presenta triangoli posti sui lati lunghi e sul lato breve sinistro e due quadrati posti sulla punta.

Tra i due mosaici la differenza principale è la correzione nel testo più recente della data di morte ( $X V$ al posto di $V X)$ e il cambio del verbo che da recessit passa a depositus (est). Dal punto di vista decorativo la corona di alloro è stata sostituita da una corona dentellala e la croce monogrammata da un quadrato posto sulla punta.

Per quanto riguarda la cronologia, basata solo su confronti stilistici interni ed esterni la basilica, si può affermare che il mosaico più antico si collochi dentro il secondo quarto del $\mathrm{V}$ secolo mentre il più recente dentro la seconda metà del V secolo, ${ }^{7}$ approssimativamente nell'ultimo decennio.

\footnotetext{
Uno studio recente afferma che l'epitaffio di Quadratianus, durante lavori di ristrutturazioni effettuati durante il VI secolo, venne ricopiato e riprodotto (Yasin 2016, 234).
} 


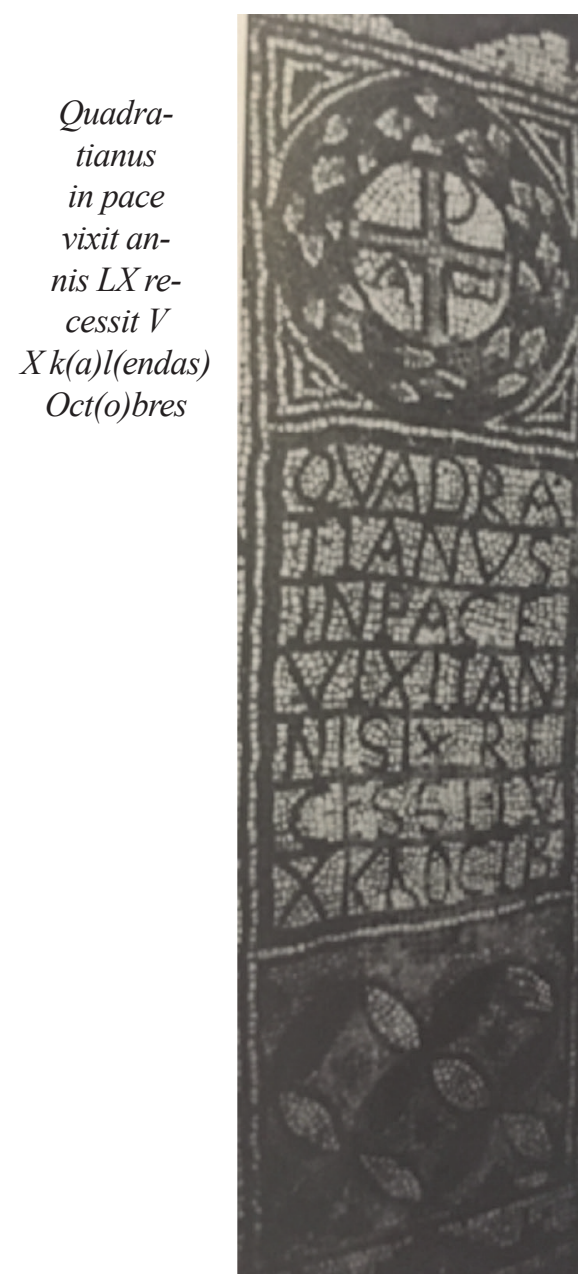

Figura 2. Primo mosaico di Quadratianus, Basilica de Uppenna, Museo dell'Enfida (Raynal 2005).

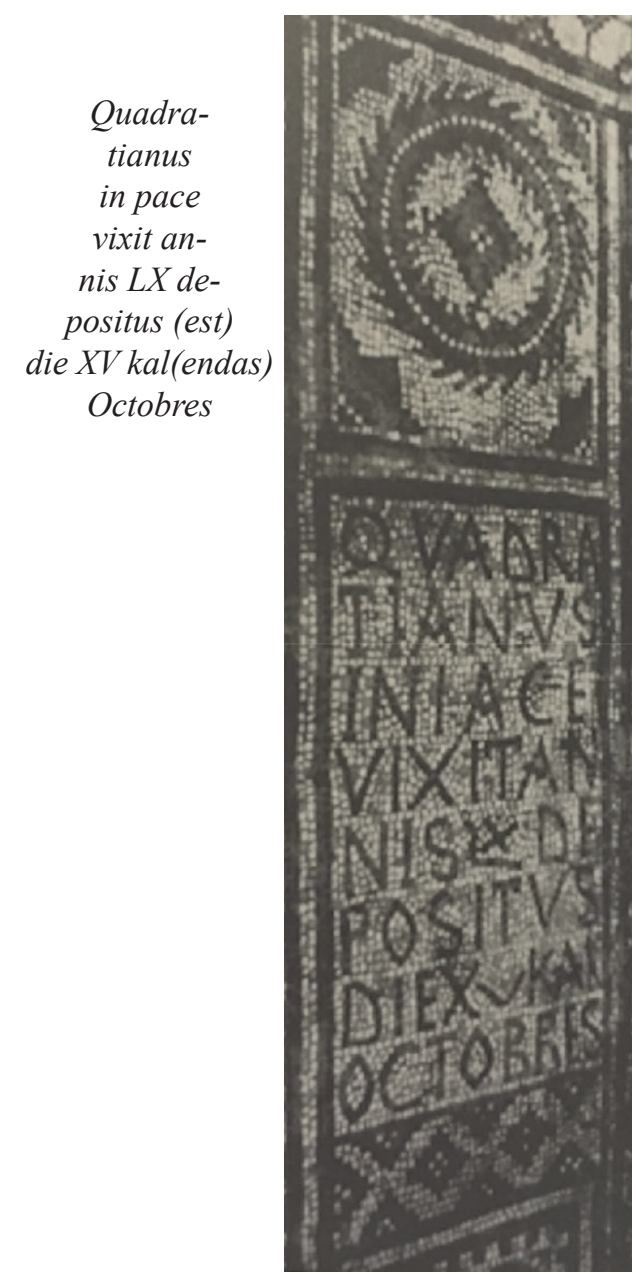

Figura 3. Secondo mosaico di Quadratianus, Basilica de Uppenna, Museo dell'Enfida (Raynal 2005).

\section{Le sepolture vescovili presenti a Uppenna}

Nella Basilica di Uppenna sono state individuate due sepolture vescovili, tutte a mosaico, rispettivamente di Baleriolus, ${ }^{8}$ databile tra la fine del V secolo e gli inizi del VI secolo, e quella di Honorius, ${ }^{9}$ databile tra il 484 e il 500 . Queste due sepolture godono di alcuni "privilegi" che non erano riservati agli altri defunti.

La sepoltura (Fig. 4) di Baleriolus si trovava nella navata centrale, contrapposta a un'iscrizione musiva ${ }^{10}$ che conteneva il ricordo di alcuni martiri (Petrus, Paulus, 


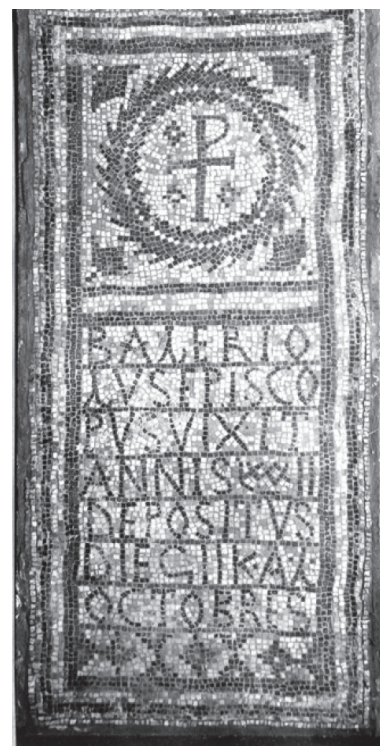

Figura 4. Mosaico di

Baleriolus, Basilica di

Uppenna, Museo dell'Enfida (EDCS).

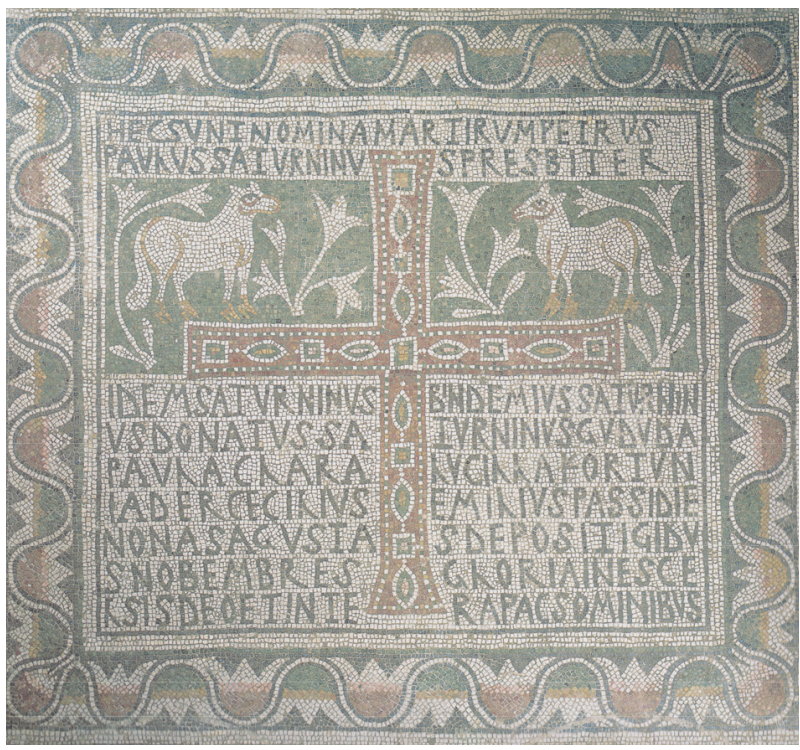

Figura 5. Iscrizione dei Martiri, Basilica di Uppenna, Museo dell'Enfida (Yacoub 1995).

Saturninus presbiter (sic!), Saturninus Bindemius, Saturninus Donatus, Saturninus Gudura, Paula, Clara, Lucilla, Fortunata, Iader, Cecilius, Emilius, Fig. 5) all'interno di una composizione musiva abbastanza ricca e nella quale trovarono posto altre due sepolture (Fig. 6): una di un bambino ${ }^{11}$ e quella di una donna. ${ }^{12}$ Probabilmente, vista la posizione delle due sepolture, contemporanee o di poco successive a quella di Baleriolus, dovevano essere due personaggi dell'élite cittadina.

La sepoltura di Honorius (Fig. 7) si trovava all'interno del vano XI, isolata rispetto alle altre sepolture ed unica all'interno del vano, vicino a una fonte battesimale e inserita all' interno di un pavimento di qualche anno anteriore (probabilmente della seconda metà del $\mathrm{V}$ secolo) che presentava una ricca decorazione. Questa decorazione era formata da uno schema geometrico con composizione a alveari di cerchi ad intreccio disegnata da ghirlande di alloro e negli spazi di risulta ottagoni (DG $234 \mathrm{f}$ var.), con rappresentazioni di uccelli all'interno dei cerchi superiori ${ }^{13}$ e nel pannello superiore una scena figurata, obliterata dalla tomba del vescovo, che però risulta parzialmente ancora leggibile. Infatti dentro questa scena erano presenti due cervi affrontati probabilmente a un kantharos, un'iconografia molto utilizzata in quel periodo. Questa scena si ricollega ad alcuni passi della Bibbia ${ }^{14}$ ed è ben documentata nell'arte musiva tardo antica: un esempio del VI secolo lo vediamo a La Skhira ${ }^{15}$ (Fig. 8); a Salona con una

\footnotetext{
Spendeu / fidelis in pace / vixit annis $V$.

Faustina / fidelis / in pace / vixit an/nis LIII.

Le figure dei cerchi inferiori risultano obliterate.

Salmi 42, 2.

Yacoub 1995, 386.
} 


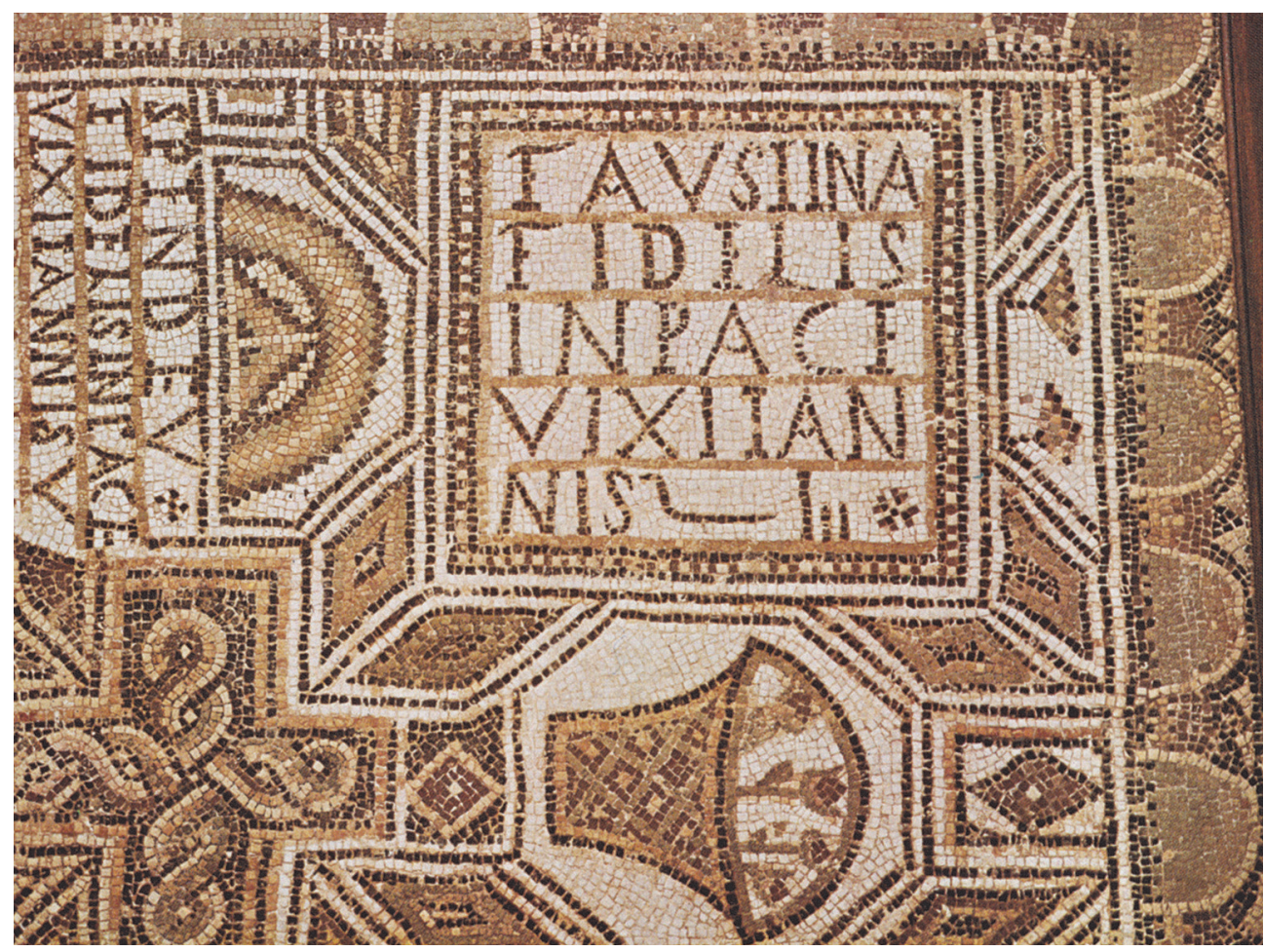

Figura 6. Mosaici di Spendeu e Faustina, Basilica di Uppenna, Museo dell'Enfida (Duval 1976).

famosa iscrizione, ${ }^{16}$ presumibilmente databile al V secolo; nell'area di Como, in Italia, datato tra il V e il VI secolo, ${ }^{17}$ e nella Macedonia Secunda come ad esempio a Edessa Longos, Akrini e Stobi, tutti pavimenti inquadrabili tra la fine del V e gli inizi del sesto secolo $^{18}$ e sempre in Nord Africa nella Basilica di El-Ounaïssia ${ }^{19}$ e infine nella Basilica di Hergla in un pavimento datato all'epoca bizantina. ${ }^{20}$

La prassi di inserire una sepoltura a mosaico di un vescovo all'interno di un programma decorativo preesistente o da sviluppare non è prerogativa della Basilica di Uppenna: almeno un'altra testimonianza l'abbiamo per quanto riguarda la Basilica di Sidi Abich. ${ }^{21}$ In questo luogo ritroviamo la tomba di Paulus episcopus ${ }^{22}$ inserita all'interno di un programma decorativo marino (Fig. 9). Il mosaico funerario non sembra essere stato messo in posa in seguito, bensì sembra essere stato concepito unitamente al mosaico "d'accompagnamento"; intorno alla copertura musiva di

16 CIL III 2673: Sic[ut cer]/vus desi/derat ad fon/tes aquarum // ita / desi/derat // anima / mea ad te / deus; Buzov 2011, 188.

17 Vanoli 2014: CO290-00005 http:/www.lombardiabeniculturali.it/reperti-archeologici/schede/CO290-00005/, consultato il 12/09/2017; Nobile De Agostini 2006, 122-123.

18 Kolaric 2016, 191-192.

19 Béjaoui 2003, fig. 45. Il mosaico con probabilità si ascrive all’interno del VI secolo.

20 Ghalia 1998, 49-57. Anche questo mosaico probabilmente si colloca nel VI secolo.

21 Yacoub 1995, 378; Baratte 2008, 226.

22 CIL VIII 23035: † Paulus / episcopus / prime se/dis provinci(a)e Mauri/tani(a)e in pa/ce requie/bit s(ub) d(ie) XV/kal(endas) / mar/tias. 


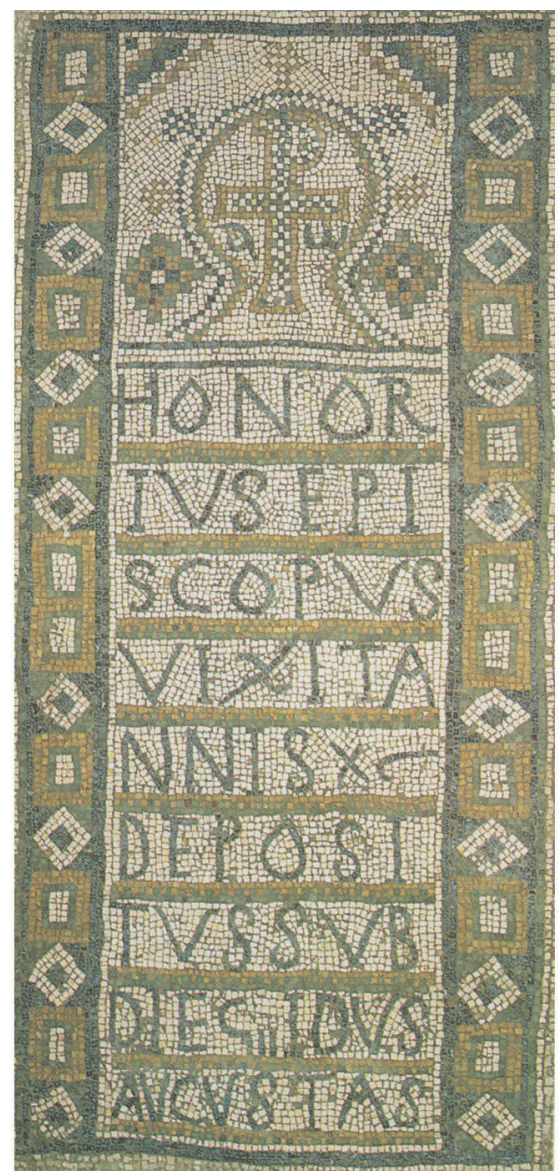

Figura 7. Mosaico di Honorius, Basilica di Uppenna, Museo dell'Enfida (Yacoub 1995).

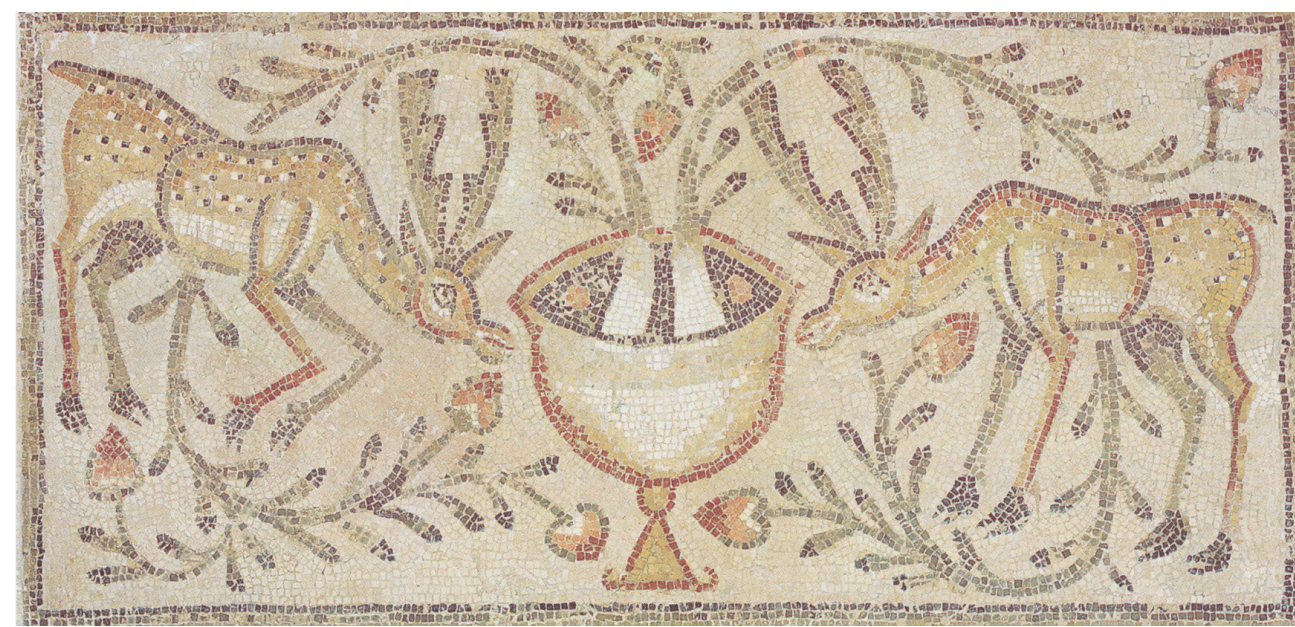

Figura 8. Mosaico con cervi da La Skhira (Yacoub 1995). 


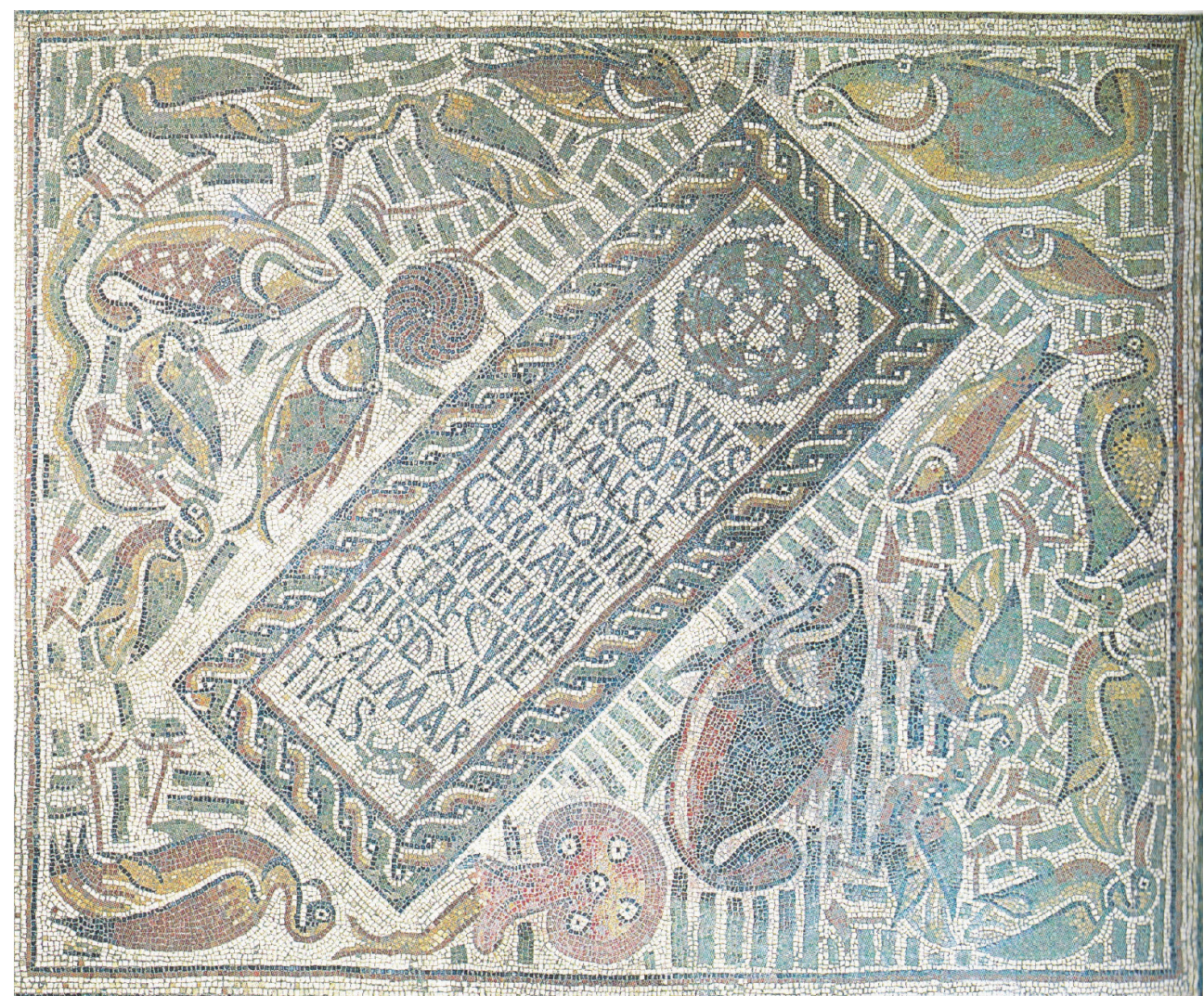

Figura 9. Mosaico di Paulus, Basilica di Sidi Abich, Museo dell’Enfida (Yacoub 1995).

Paulus ruotano pesci e gru, e le prove che il mosaico funerario fosse stato realizzato in contemporanea con il tessellato con decorazione musiva sono diverse: i colori delle tessere sono gli stessi utilizzati sia per il mosaico funerario che per il tessellato, la dimensione delle tessere sembra essere uguale ${ }^{23}$ e in ultimo si devono notare le fasce di tessere di colore verde utilizzate per realizzare le onde, che riempiono gli spazi vuoti, le quali dimostrano che i due mosaici sono stati concepiti in maniera unitaria. Interessante è anche il fatto che Paulus non fosse il vescovo della zona, ma anzi la sua sede vescovile si trovasse molto lontana dal luogo di sepoltura.

\section{Una basilica donatista?}

Intorno a questa domanda D. Raynal produce una lunga disgressione ${ }^{24}$ affermando che la Basilica di Uppenna, pur trovandosi nella zona costiera della Tunisia orientale e non certamente il cuore dei donatisti, ${ }^{25}$ fosse uno dei monumenti più importanti di

23 Questo porta a pensare che fosse la stessa identica bottega ad aver messo in posta entrambi i tessellati.

24 Raynal 2005, 759-792. Sul tema aveva già parlato in Raynal 1973, 33-72.

25 Si veda anche Février 1996, 699-711. 


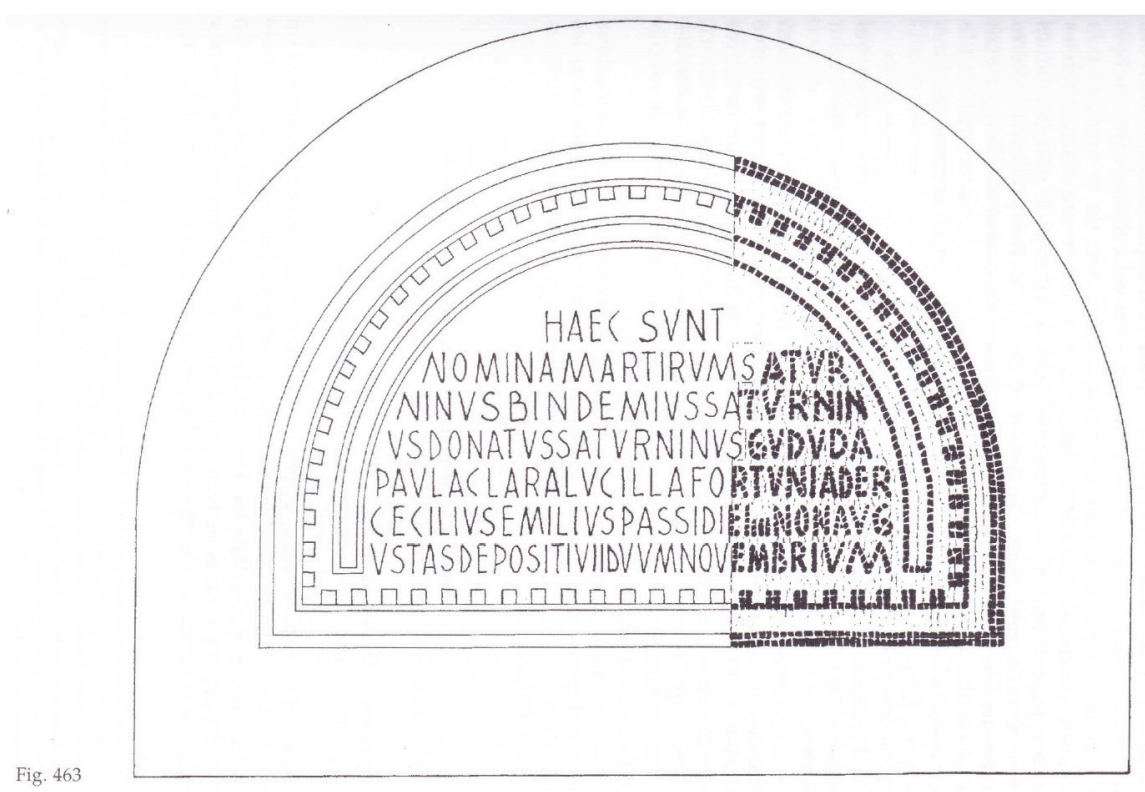

Figura 10. Prima iscrizione dei Martiri, ipotesi ricostruttiva (Raynal 2005).

questa fede. ${ }^{26}$ Senza dubbio ci sono delle tracce che potrebbero far pensare che questa basilica fosse una roccaforte donatista. Un esempio è l'acclamazione ritrovata nell'epitaffio musivo di una sepoltura che recita: (H)ic qui digni sunt sic accipiant. ${ }^{27}$ Indubbiamente l'acclamazione è evocativa dello spirito Donatista, ${ }^{28}$ grazie alla similitudine con espressioni certamente donatiste conosciute in Nord Africa. ${ }^{29}$ Però un'espressione simile sarebbe potuta essere utilizzata indistintamente da Donatisti e non all'interno della polemica retorica del IV e V secolo. Un'altra argomentazione riportata da Raynal riguarda i martiri ricordati nell' iscrizione ritrovata in un'absidiola ${ }^{30} \mathrm{e}$ addossata, come ricordato, alla sepoltura di Baleriolus:

Hec sunt nomina martirum Petrus / Paulus Saturninus presbiter / idem Saturninus Bindemius Saturnin/us Donatus Saturninus Gududa / Paula Clara Lucilla Fortun(ata) / Iader Cecilius Emilius passi die / Nonas Augustas depositi VI Nobembres gloria in esce/lsis Deo et in ter(r)a (h)ominibus.

26 Raynal 2005, 785.

27 Raynal 2005, 490-492.

28 Probabilmente scettico era N. Duval che scrive: "La liste des martyrs vénérés a justifié, aux yeux de certains, une appartenance donatiste" (Duval 1989, 396).

29 ILCV 2435a: Sanctorum se/des domu(s) domi/ni qui pure peti/t ac(c)ipit; Monceaux 1907, 276-277.

30 Il mosaico era sovrapposto a un altro più antico (Fig. 10), di forma semicircolare sempre in tessellato. È ormai assodato che il testo dei due mosaici differisse in alcune parti: Y. Duval $(1982,62)$ sostiene che in particolare la prima linea dell'iscrizione dovesse essere differente, ad esempio, senza l'invocazione dei primi tre nomi. 
Raynal ipotizza che il terzo personaggio, Saturninus presbiter, menzionato nell'iscrizione fosse il leader dei martiri d'Abitina. ${ }^{31}$ Ipotizzando fosse vero, gli stessi, non sarebbero stati una proprietà esclusiva dei donatisti.

In effetti, però, la tesi di Raynal è affascinante. Lo studioso interpreta l'iscrizione martiriale come una rappresentazione della Chiesa ponendo in comunicazione i Santi Pietro e Paolo con Saturnino. ${ }^{32}$ Anche lo studioso A. Rossi concorda con questa tesi ${ }^{33}$ notando come i primi tre nomi, appunto Pietro, Paolo e Saturnino, facciano da "guide del gruppo". ${ }^{34}$ Ed è simbolico il fatto che il vescovo Baleriolus fosse stato interrato proprio prospicente a questa epigrafe musiva, forse un indizio della fede donatista di quest'ultimo.

\section{Ipotesi sull'identificazione del defunto}

Come abbiamo visto, l'inserimento di una tomba all'interno di un programma decorativo musivo precedente o contemporaneo era un trattamento riservato a persone illustri e in particolar modo ai vescovi. ${ }^{35}$

E logico dunque pensare che Quadratianus dovesse godere di una qualche forma di rispetto e reverenza per aver ricevuto una sepoltura lontana dall'affollamento delle tre navate, in un vano isolato e interamente decorato a mosaico.

È possibile forse pensare che il nostro defunto fosse quel Quadratianus episcopus Sicilibbensis presente al Concilio di Cartagine nel 411? Il vescovo di Sicilibba era donatista $^{36}$ e se le ipotesi di alcuni studiosi fossero corrette, come precedentemente accennato, sulla possibile fede donatista della Basilica di Uppenna non renderebbero strana la sua presenza.

Se ipotizzassimo che Quadratianus episcopus Sicilibbensis fosse lo stesso personaggio sepolto ad Uppenna, dobbiamo tenere in conto dell' età del defunto: l'uomo ha 60 anni e se fosse stato ordinato vescovo intorno ai 30 anni $^{37}$ al momento del Concilio di Cartagine era stato, presumibilmente, appena ordinato vescovo. Se la datazione del mosaico più antica dovesse essere vera, dunque intorno al secondo quarto del V secolo, sembrerebbe plausibile che si possa parlare della stessa persona.

Sulla base degli elementi sopraindicati viene da chiedersi del perché il vescovo di Sicilibba si trovasse ad Uppenna, dunque nella diocesi Oppennensis.

Come si sa, dopo lunghi assedi e guerre, i Vandali vennero legittimati dall'Imperatore Valentiniano III, grazie a un trattato di pace, a insediarsi in Africa, così come racconta Prospero D’Aquitania: ${ }^{38}$

\footnotetext{
31 Tesi non accettata da tutti, si veda: Duval 1982, 686. Sui martiri si veda inoltre: Beschaouch 1976, 255-266.

32 Raynal 2005, 721.

33 Il quale non è da solo perché anche Dalvit recentemente sposa questa ipotesi (Dalvit 2013, 41).

34 Rossi 2013, 271-272.

35 Penso di poter escludere l'ipotesi di un vano dedicato a sepolture familiari. Infatti il pavimento geometrico precedente la sepoltura fu rimaneggiato solo ed esclusivamente per accogliere la sepoltura, fatto che già di per sé portò un costo non indifferente. Se il vano fosse stato concepito per sepolture familiari gli artigiani non si sarebbero adoperati per rendere il pavimento geometricamente corretto.

36 Mandouze 1982, 934.

37 Una pratica comunque che non stupisce, come si evince ad esempio da CIL XIII 5407: [...] Silvester / episcop (us) / qui vixit in pace / ann(os) XXXXVII et / mansit in episc(opatu) / ann(os) XXII[- - -].

38 Prosp. Epit. Chron. 9.427-442.
} 
[a.427] Gens Wandalorum ab Hispania ad Africam transit; [a. 435] Pax facta cum Vandalis data eis ad habitandum Africae portione; [a. 442] Cum Gisirico ab Augusto Valentiniano pax confirmata et certis spatiis Africa inter utrumque divisa est.

Ma si legge anche che:

[a. 439] Aetio rebus quae in Gallia componebantur intento Gisiricus de cuius amicitia nihil metuebatur, Carthaginem dolo pacis invadit omnesque opes eius excruciatis diverso tormentorum genere civibus in ius suum vertit, nec ab ecclesiarum despoliatione abstinens, quas et sacris vasis exinanitas et sacerdotum admistratione privatas non iam divini cultus loca, sed suorum esse iussit habitacula, in universum captivi populi ordinem saevus, sed praecipue nobilitati et religioni infensus, ut non discerneretur, hominibus magis an deo bellum intulisset. Hanc autem captivitatem Carthago subiit anno, postquam Romana esse coeperat, quingentesimo et octogensimo et quinto.

Questo porta il clero, che si oppone all'arianesimo, a trovare rifugio in sedi più sicure, e probabilmente è in questo periodo che il nostro Quadratianus, trovandosi privato della sua chiesa, domandò asilo alla diocesi di Oppennensis. Di avviso contrario è Y. Modéran che infatti afferma che i vescovi, venissero sì esiliati, ma a pochi $\mathrm{km}$ dalla propria sede episcopale. ${ }^{39}$ In realtà abbiamo però testimonianza di come Fulgenzio da Ruspe venne espulso ben oltre i confini africani. ${ }^{40}$ Vittore de Vita infatti divide $i$ vescovi esuli in due gruppi: coloro che vennero espulsi e rimasero nella stessa Africa, e coloro che vennero esiliati in Corsica. ${ }^{41}$

Non sarebbe neanche l'unica sepoltura vescovile lontana dalla sede del defunto. Prima si accennava al mosaico funerario di Paulus ritrovato nella Basilica di Sidi Abich. ${ }^{42}$ I personaggi provenivano dalla Mauretania, Paulus, e dalla Proconsolare Quadratianus, dunque non sembrerebbe così strano che entrambi i vescovi si trovassero lontani dalla propria sede vescovile quando sopraggiunse loro la morte.

La mancanza però della titolatura di episcopus stride con questa ipotesi. Possiamo forse pensare che l'assenza della titolatura sia da implicare a una mancanza del committente, che nel momento di realizzare l'epitaffio non si è sincerato che fosse presente la carica clericale del defunto, o chissà, perché Quadratianus era un personaggio poco conosciuto dagli artigiani. Ulteriore interpretazione potrebbe trovarsi nell'intendere che il defunto, trovandosi lontano dalla propria sede episcopale, venne trattato, al momento della sepoltura, come un privatus. Altresì si potrebbe supporre che sia stato omesso volutamente il termine episcopus per evitare eventuali ritorsioni o trafugamento di spoglie. Quest'ultima affermazione potrebbe essere avvalorata anche dal fatto che la carica vescovile viene inserita negli epitaffi della Basilica di Uppenna a partire dalle ultime decadi del V secolo prime decadi del VI secolo (i già ricordati Baleriolus e Honorius) quando il Regno dei Vandali era entrato in una fase di inesorabile declino.

\footnotetext{
Modéran 1993, 185.

Vita Fulgenti 17.87.

Modéran 1993, 166.

Le due basiliche si trovano a pochi chilometri di distanza.
} 
Interessante è anche notare come la bottega che mise in posa il mosaico del vescovo Baleriolus è senza dubbio alcuno la stessa che fabbricò il secondo mosaico di Quadratianus. Se in quegli anni la Basilica di Uppenna fosse stata nelle mani dei donatisti, come sembra suggerirci la sepoltura di Baleriolus, vicina all'epigrafe con l'iscrizione in ricordo dei martiri, si può supporre che il vescovo Baleriolus avesse deciso di intraprendere una ristrutturazione degli apparati decorativi della Basilica che interessò anche la tomba di Quadratianus.

Quella qui esposta è un'ipotesi, probabilmente azzardata e supportata da prove, o meglio indizi, non sempre forti; in ogni caso è da evidenziare l'importanza del personaggio ivi interrato, tralasciando quale potesse essere la sua carica istituzionale. Ė infatti indubbia la volontà, a distanza di decenni, di perpetuare la memoria del defunto, una pratica certamente non usuale se non per persone dalla spiccata levatura sociale. Che fosse o meno un episcopus rimarrà, probabilmente, sempre un dubbio, ma certa rimane la sua oggettiva importanza all'interno della comunità.

\section{Conclusioni}

Ricapitolando, gli indizi che ci portano a pensare che Quadratianus fosse un personaggio di spicco del clero, probabilmente un vescovo, sono:

- Sepoltura in un'area isolata della basilica, lontana da quelle degli altri fedeli e dei vescovi della diocesi di Uppenna;

- Sepoltura curata, con mosaico funerario inserito in un programma decorativo più elaborato rispetto ai normali fedeli, simile al trattamento riservato ai vescovi nella stessa basilica;

- Rifacimento, a distanza di una generazione, della sepoltura;

- Presenza di un Quadratianus episcopus Sicilibbensis, di fede donatista, nel Concilio di Cartagine del 411;

- Possibile frequentazione da parte dei donatisti della Basilica di Uppenna.

Se questa ipotesi dovesse essere corretta, ci troviamo di fronte, non soltanto a una sepoltura certamente privilegiata (che lo sarebbe anche nel caso che Quadratianus non fosse un vescovo), ma anche a una traccia, un resto, della persecuzione operata dai Vandali ai danni degli ortodossi.

Purtroppo la mancanza di dati certi, che uno studio del materiale avrebbe potuto dare, ci porta ad usare la massima cautela e di muoverci, comunque, nell'ambito delle ipotesi. Rimane certo il fatto che Quadratianus fosse un personaggio certamente importante all'interno della comunità, visto che la sua sepoltura ricalca la fisionomia di personaggi molto importanti del clero di Uppenna.

\section{Bibliografia}

Balmelle, C. - Blanchard Lemée, M. - Christophe, J. - Darmon, J. P. - Guimier-Sorbets, A. M. - Lavagne, H. - Prudhomme, R. - Stern, H.

(1985): Le décor geometrique de la mosaïque romaine. I. Répertoire graphique et descriptif des compositions linéaires et isotropes, Paris $(=D G)$. 
(2002): Le décor geometrique de la mosaïque romaine. II. Répertoire graphique et descriptif des décors centrés, París $(=D G)$.

Baratte, F. (2008): "Les èvêques et leur sépulture en Afrique. Les données archéologiques", [in] Lieux de culte: aires votives, temples, églises, mosquées. IX colloque international sur l'histoire et l'archéologie de l'Afrique du Nord antique et médievale (Tripoli, 19-25 fèvrier), Paris, 225-236.

Béjaoui, F. (2003): "Les mosaïques funéraires de Sufetula (Sbeïtla) et de Thagamuta (Ksar el Barout)", [in] Actes du VIII colloque international sur l'histoire et l'archéologie de l'Afrique du Nord (Tabarka, 8-13 mai 2000), Tunis, 303-333.

Beschaouch, A. (1976): "Sur la localization d'Abitina, la cité des célèbres martyrs africains", CRAI 1976, 255-266.

Buzov, M. (2011): “The Early Christian Mosaics with Inscription in Croatia”, [in] M. Sahin (ed.), Mosaics of Turkey and Parallel Developments in the Rest of the Ancient and Medieval World: Questions of Iconography, Style and Technique from the Beginnings of Mosaic until the Late Byzantine Era, Istanbul, 171-192.

Dalvit, M. (2013): Ecclesia martyrum. Analisi del corpus martirologico donatista, Tesi di Dottorato, Università degli Studi di Padova.

Dhiel, E. (1925-1931): Inscriptiones Latinae Christianae Veteres, Berlin, 3 vol. (=ILCV).

Duval, N.

(1976): La mosaïque funéraire dans l'art paléochrétien (=Antichitá, Archeologia, Storia dell'Arte 3), Ravenna.

(1989): "L'évêque et la cathédrale en Afrique du Nord", [in] Actes du XI congrès international d'archéologie chrétienne. Lyon, Vienne, Grenoble, Genève, Aoste (21-28 septembre 1986), (=Publications de l'École française de Rome 123), Roma, 1989, vol. 2, 345-399.

Duval, Y. (1982): Loca Sanctorum Africae. Le culte des Martyrs en Afrique du IV au VII siecle (=Publications de l'École française de Rome 58), Roma, 2 vols.

Février, P. A. (1996): “Toujours le donatisme. À quand l'Afrique? (Remarques sur l'Afrique à la fin de l'Antiquité, à propos du livre de E. Tengström)", [in] La Méditerranée de PaulAlbert Février [recueil d'articles], (=Publications de l'École française de Rome 225) Roma, 699-711.

Ghalia, T. (1998): Hergla et les mosaïques de pavement des basiliques chrétiennes de Tunisie: Plan, décor et liturgie, Tunis.

Kolarik, R. (2016): "A study of mosaic workshop - Macedonia Secunda and Epirus Nova", [in] L. Neira (ed.), Estudios sobre mosaicos antiguos y medievales. Actas del XIII Congreso AIEMA Madrid 14-18 de septiembre de 2015 (=L'Erma di Bretschneider. Hispania Antigua. Serie Arqueológica 6), Roma, 189-198.

Mandouze, A. (1982): Prosopographie chrétienne du Bas-Empire, 1. Prosopographie de l'Afrique chrétienne (303-533), Paris.

Modéran, Y. (1993): "La chronologie de la Vie de saint Fulgence de Ruspe et ses incidences sur l'histoire de l'Afrique vandale", Mélanges de l'École française de Rome 105/1, 135-188.

Monceaux, P. (1907): Enquête sur l'épigraphie chrétienne d'Afrique. IV. Martyrs et reliques, Paris.

Nobile De Agostini, I. (2006): La sezione romana del Museo archeologico di Como. Guida all'esposizione, Como.

Quattrocchi, L. (2017): Los mosaicos funerarios de Túnez, España e Italia. Siglos III-VII, Tesi di dottorato, Universidad Carlos III de Madrid - Università di Sassari. 
Raynal, D.

(1973): "Culte de Martyrs et propagande donastiste à Uppenna", Cahiers de Tunisie 8182, 33-72.

(1982): “La Basilique d'Uppenna: de la periode vandale a l'époque byzantine. Interpretation des resultats des fouilles de 1904-1906", Africa 7-8, 179-284.

(1995): “Une Église persécutée: l'Église donatiste. Approches historique et archéologique", Cahiers de Tunisie 169-170, 63-86.

(2005): Archéologie et histoire de l'Église d'Afrique. Uppenna II. Mosaïques funéraires et mémoire des martyrs, Toulouse.

Rossi, A. (2013): Muscae Moriturae Donatistae Circumvolant, Torino.

Stevens, S. T. (2007): "Of martyrs and mosaics: two Early Christian churches at Sidi Jdidi (Aradi) and Henchir Chigarnia (Uppenna)", JRA 20, 689-696 (https://doi.org/10.1017/ S1047759400006188).

Yacoub, M. (1995): Splendeurs des mosaïques de Tunisie, Tunis.

Yasin, A. M. (2016): "Beyond spolia. Architectural Memory and Adaptation in the Churches of Late Antique North Africa", [in] S. T. Stevens - J. P. Conant (eds.), North Africa under Byzantium and Early Islam, Washington, 215-236. 